\title{
PERFIL DE ENFERMEIROS FLUMINENSES DA ESTRATÉGIA DE SAÚDE DA FAMÍLIA PARTICIPANTES DE UM CURSO DE ESPECIALIZAÇÃO
}

Magda Guimarães de Araujo Faria ${ }^{1}$, Sonia Acioli², Cristiane Helena Gallasch ${ }^{3}$

Objetivo: analisar o perfil dos enfermeiros fluminenses atuantes na Estratégia de Saúde da Família on-line participantes de curso de especialização em saúde da família. Metodologia: estudo de abordagem quantitativa, com desenho transversal e observacional, não experimental e de tipo descritivo. A coleta de dados foi feita com 131 sujeitos que responderam ao questionário on-line. Para o tratamento dos dados foi utilizado o programa Statistical Package for the Social Sciences. Resultados: o perfil de enfermeiros fluminenses atuantes na ESF é composto por maioria de mulheres, com faixa etária inferior a 40 anos, oriundas de instituições particulares de ensino e com menos de 10 anos de conclusão da graduação. Conclusão: a ESF é grande empregador das novas gerações da Enfermagem, remetendo à necessidade da adequação das unidades de ensino à formação desses profissionais, considerando a ampliação dessa cobertura.

Descritores: Estratégia Saúde da Família, Enfermagem em Saúde Comunitária, Enfermagem em Saúde Pública.

\section{PROFILE OF FLUMINENSE NURSES OF FAMILY HEALTH STRATEGY PARTICIPANTS OF SPECIALIZATION COURSE}

Objective: analyze the profile of Rio de Janeiro nurses who working in the Family Health Strategy on-line participants of the specialization course in family health. Methodology: quantitative approach study with cross-sectional and observational design, not experimental and descriptive type. Data collection was performed with 131 subjects who answered the online questionnaire. For data analysis was used Statistical Package for the Social Sciences Results: the profile of working fluminense nurses in the ESF consists of most women, aged under 40 years, coming from private institutions of education and less than 10 years of completed the undergraduation degree. Conclusion: the ESF is a large employer of the newer generation of nurses. There is a need to adequate the educational facilities for the training of nurses prepared to work in public health.

Descriptors: Family Health Strategy, Community Health Nursing, Public Health Nursing.

\section{PERFIL DE ENFERMEROS FLUMINENSES DE LA ESTRATEGIA DE SALUD FAMILIAR PARTICIPANTES DE UN CURSO DE ESPECIALIZACIÓN}

Objetivo: analizar el perfil de las enfermeras de Río de Janeiro que trabajan en el Estregia de Salud Familiar on-line del curso de especialización en salud de la familia. Metodología: estudio cuantitativo con diseño transversal y observacional, no experimental y descriptivo. La recolección de datos se realizó con 131 sujetos que respondieron el cuestionario en línea. Para el tratamiento de los datos se utilizó el programa Statistical Package for the Social Sciences Resultados: se observó que el perfil de las enfermeras de Río de Janeiro que trabajan en la ESF consiste principalmente de mujeres con edades inferiores a 40 años, proveniente de instituciones privadas de educación, y menos de 10 años de la finalización de la graduación. Conclusión: el ESF es el principal empleador de las nuevas generaciones de enfermería, lo que lleva a la necesidad de adecuación de las instalaciones educativas para la formación de enfermeras preparadas para trabajar en la salud pública.

Descriptors: Estrategia de Salud Familiar, Enfermería en Salud Comunitaria, Enfermería en Salud Pública.

1Enfermeira. Doutora. Professora da Faculdade de Enfermagem da Universidade do Estado do Rio de Janeiro. ${ }^{2}$ Enfermeira. Doutora. Professora da Faculdade de Enfermagem da Universidade do Estado do Rio de Janeiro.

${ }^{3}$ Enfermeira. Doutora. Professora da Faculdade de Enfermagem da Universidade do Estado do Rio de Janeiro. Email: cristiane.gallasch@gmail.com 


\section{INTRODUÇÃO}

A Estratégia de Saúde da Família (ESF) pode ser considerada, hoje, um dos principais cenários da saúde pública brasileira, não apenas pela possibilidade de intervir junto à população na perspectiva da vigilância da saúde, mas também por conta da magnitude do quantitativo de trabalhadores nela inseridos.

Até o ano de 1996, a ESF, chamada de Programa de Saúde da Família, possuía uma estratégia de expansão de cobertura e, a partir do ano de 1996, começou a ser vista como uma reorientação do modelo assistencial, sobretudo à atenção básica de saúde ${ }^{(1)}$.

Assim, observa-se a intensa ampliação da cobertura das ESF a partir do início dos anos 2000. Ainda não é possivel dizer que o percentual da população atendida pela ESF é o ideal, entretanto, caminha-se a passos largos para tal objetivo. Nos últimos 15 anos, o Brasil triplicou a cobertura. No Rio de Janeiro, o número de equipes vinculadas ao SF aumentou $500 \%{ }^{(2)}$.

A ampliação da ESF permitiu aumentar o número de indivíduos atendidos. Em contrapartida, gerou uma necessidade de qualificação que, devido a fatores como a extensa jornada de trabalho e a interiorização das unidades, tornava-se uma atividade de dificil implementação.

Neste sentido, é cada vez mais comum o uso de propostas alternativas ao ensino presencial, voltados à qualificação profissional, como por exemplo, a educação a distância. A integração das atividades de educação permanente com a educação a distância permite não apenas a qualificação profissional, mas a autogestão do conhecimento e, a eliminação de barreiras temporais e geográficas ${ }^{(3)}$.

Assim sendo, esta pesquisa propõe analisar o perfil dos enfermeiros fluminenses atuantes na ESF, participantes de um curso de especialização on-line em saúde da família.

\section{METODOLOGIA}

O presente estudo é de abordagem quantitativa, de desenho transversal e observacional, de delineamento de pesquisa não experimental e de caráter descritivo.

Participaram do estudo 131 sujeitos do Curso de Especialização em Saúde da Família ministrado on-line, que atenderam aos critérios de inclusão: ser graduado em Enfermagem; ter finalizado todas as etapas do curso, ou seja, oito disciplinas obrigatórias, três disciplinas obrigatórias transversais, duas disciplinas eletivas, o TCC e a prova final de avaliação do conteúdo. Responderam o questionário 66 participantes do Curso.

Como instrumento de coleta de dados foi utilizado um questionário com 27 questões sobre dados pessoais (sexo, idade e motivação para o curso) e profissionais (tempo de formação, tempo de atuação e área de especialização). Optouse pelo uso do questionário por ser um relevante instrumento frente à obtenção de informações em uma pesquisa e permitir detectar opiniões, sentimentos e interesses dos sujeitos em uma pesquisa(4).

O questionário foi disponibilizado aos sujeitos via internet, com uso do aplicativo de questionários on-line denominado SurveyMonkey. A dinâmica ocorreu com o envio de um e-mail contendo um link de acesso para o preenchimento do formulário.
O questionário foi enviado aos sujeitos da pesquisa por meio de correio eletrônico. Foram, ao total, seis mensagens coletivas entre os meses de maio e julho de 2014. Apenas alunos que não haviam respondido o questionário recebiam as mensagens. Além disso, com o intuito de aumentar a adesão à pesquisa, foi enviada, também, uma mensagem via torpedo eletrônico para o celular dos alunos que, em julho, ainda não haviam respondido o questionário. As tutoras das respectivas turmas também enviaram mensagens coletivas aos alunos, lembrando-os da participação na pesquisa.

A análise e tratamento dos dados foram realizadas a partir da utilização do programa StatisticalPackage for the Social Sciences (SPSS), já integrado à plataforma SurveyMonkey. Os dados foram quantificados, tabulados, analisados e apresentados em gráficos.

O projeto foi aprovado pelo Comitê de Ética em Pesquisa da Sub-Reitoria de Pós-Graduação e Pesquisa da UERJ, sob o parecer $\mathrm{n}$ ㅇ 579.747.

\section{RESULTADOS}

Dos 131 individuos selecionados para participar da pesquisa, 66 (50,3\%) responderam o questionário. A porcentagem obtida é muito superior à média de $25 \%$ do índice de devolução de questionários ${ }^{(5)}$

Como já era esperado, a grande maioria dos participantes da pesquisa foi do sexo feminino. O percentual de profissionais do sexo feminino corresponde a $84 \%$, enquanto os profissionais do sexo masculino atingem $16 \%$ dos registros.

Observou-se, ainda, uma alta predominância de profissionais abaixo dos 40 anos, aproximadamente $86 \%$ do total dos respondentes. Esse resultado converge para a proporção encontrado em outra experiência, em que foi observado que, aproximadamente, $55 \%$ dos enfermeiros brasileiros possuem menos de 40 anos de idade ${ }^{(6)}$.

Percebeu-se, nesta pesquisa, que 95\% dos egressos possuiam até dez anos de formados e, que $70 \%$ dos profissionais concluíram o ensino superior em uma instituição privada.

Além disso, é notória a grande preocupação dos egressos em relação à sua formação acadêmica. Aproximadamente 64\% já possuíam algum certificado de especialista antes de concluir o curso analisado, conforme Gráfico 1 .

Gráfico 1 - Área de especialização dos enfermeiros fluminenses atuantes na ESF - Rio de Janeiro, 2015

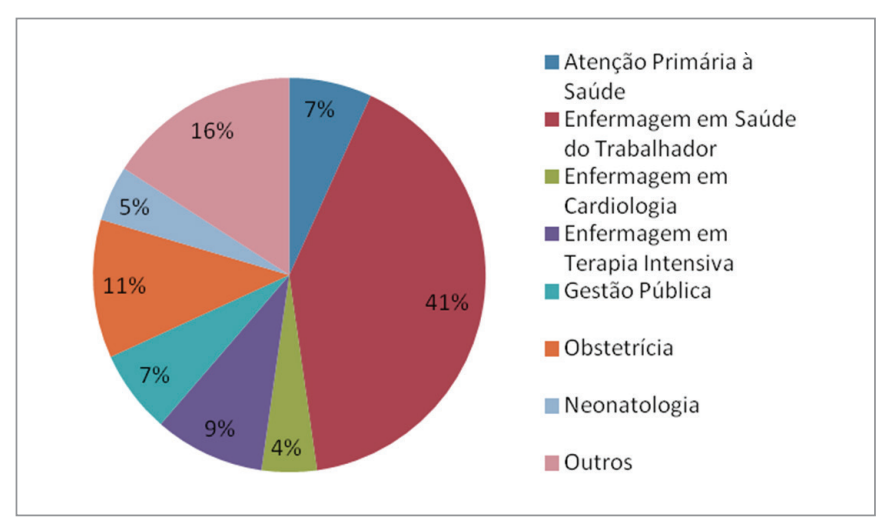


Enfatiza-se que os profissionais participantes desta pesquisa são ou já foram trabalhadores da Assistência Primária de Saúde (APS), entretanto, observou-se um baixo percentual de enfermeiros que já haviam realizado o curso de especialização em Saúde da Família. Imagina-se que esse fato ocorreu porque, para ser selecionado como aluno do curso analisado, o estudante deveria ser recomendado pelo gestor do município e, certamente, não é prioridade para a gestão indicar um aluno que já é especialista na área de concentração do curso. Chama a atenção, ainda, para o alto percentual de profissionais especialistas em enfermagem do trabalho.

Foi analisada ainda a motivação para ingressar no curso estudado. Nesta pesquisa, percebeu-se que os enfermeiros concluintes escolheram o curso de especialização em Saúde da Família motivados, essencialmente, pela necessidade pessoal de aprimoramento. Além disso, 50\% dos respondentes indicaram a Saúde da Família como área de escolha para crescimento profissional e certificação de especialista. O Gráfico 2 complementa essa informação.

Gráfico 2 - Análise da atuação dos enfermeiros fluminenses atuantes na ESF. Rio de Janeiro, 2015

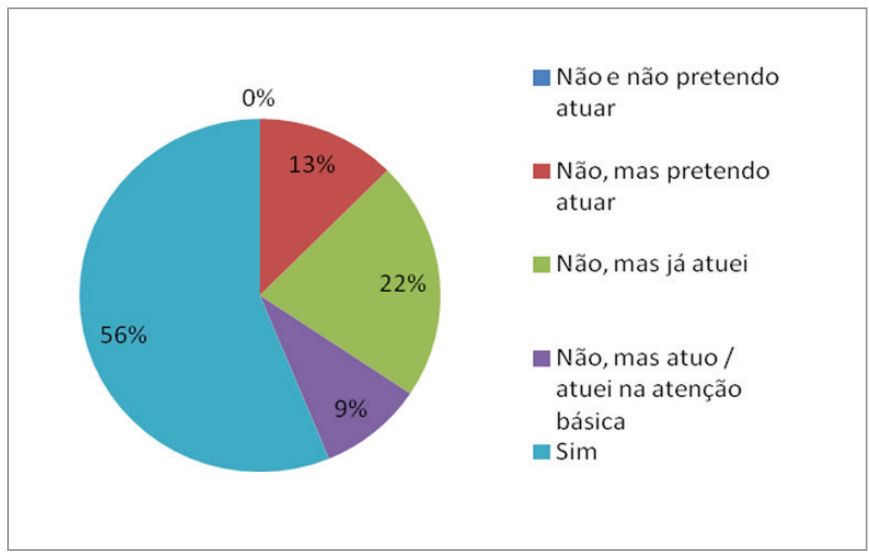

Observa-se que $22 \%$ dos profissionais não atuavam mais na ESF. Baseando-se na duração anual do curso, considera-se este um percentual muito elevado.

Verificou-se, ainda, que $72 \%$ dos enfermeiros atuam na ESF há mais de um ano. O Gráfico 3 apresenta o percentual de enfermeiros conforme o tempo de atuação na ESF.

Gráfico 3 - Anos de atuação dos enfermeiros fluminenses em ESF- Rio de Janeiro, 2015.

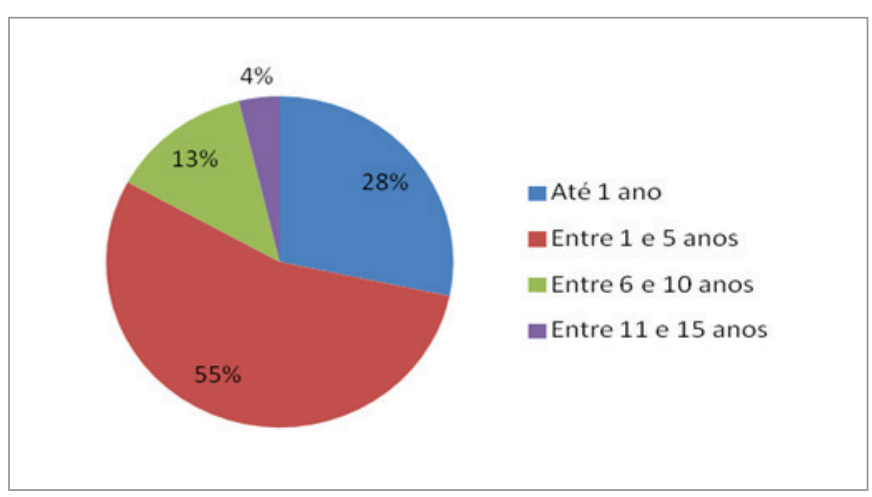

\section{DISCUSSÃO}

A presença do maior quantitativo de mulheres na força de trabalho da enfermagem da ESF fluminense é um achado que reafirma o perfil e o histórico das práticas de Enfermagem. Além disso, está em consonância com outras pesquisas realizadas com profissionais da mesma área $a^{(6-9)}$.

Sobre o perfil demográfico do enfermeiro da ESF no Rio de Janeiro, outros estudos indicam resultados semelhantes. No estado do Mato Grosso, a faixa etária entre 20 e 30 anos é a que engloba o maior número de profissionais, entretanto, o índice não chegou aos $30 \%$, enquanto que, no presente estudo, ultrapassou os $50 \%$ (8)

Estima-se que a concentração de profissionais jovens a ESF está relacionada, sobretudo, à ampliação nacional da cobertura e, consequentemente à disponibilidade de vagas de emprego. Nos últimos 10 anos, houve um aumento de $61 \%$ no número total de habitantes atendidos pela $\operatorname{ESF}^{(2)}$.

A ampliação da cobertura da ESF resulta no aumento do número de equipes e, também, de enfermeiros. Assim, abre-se um nicho de trabalho para os profissionais jovens, com poucos anos de experiência e que ainda não se estabeleceram no mercado de trabalho.

Nesse sentido, observa-se a equivalência entre a recente formação dos enfermeiros fluminenses aos achados em outros estudos da APS. Em Uberaba-MG, 73\% dos enfermeiros atuantes no SF graduaram-se nos últimos cinco anos ${ }^{(10)}$. Já em Mato Grosso, o índice de enfermeiros atuantes na ESF e graduados nos últimos cinco anos é de $48,1 \%{ }^{(8)}$.

Assim como nesta pesquisa, outros estudos apontam para a predominância da formação particular do enfermeiro fluminense atuante na $\operatorname{ESF}^{(7)}$. Apesar da constância deste perfil, observa-se uma grande modificação quando este profissional é comparado ao enfermeiro atuante na ESF na década passada. No ano de 2000, o percentual de enfermeiros da ESF formados em instituições privadas não ultrapassava $30 \%$ (11). Meia década mais tarde, os números continuavam neste mesmo patamar, à exceção das regiões sul e sudeste, onde a empregabilidade de enfermeiros da rede privada de ensino chegava a $40 \%{ }^{(12)}$.

Acredita-se que esta modificação, ao passar dos anos, tenha ocorrido em função do aumento da demanda, que era - e ainda é - incompatível com o número de profissionais inseridos no mercado, oriundos das instituições públicas de ensino.

O número de instituições privadas que oferecem cursos de graduação em enfermagem é equivalente, hoje, a $85 \%$ do total. Em números absolutos, das 929 instituições que oferecem o curso, apenas 142 são públicas. No Rio de Janeiro, as faculdades privadas de enfermagem correspondem a $92 \%$ dos cursos em funcionamento(13)

Sobre a formação complementar, percebeu-se um aumento do percentual de trabalhadores especialistas em alguma área. Em pouco mais de uma década, o quantitativo de enfermeiros especialistas atuantes na ESF aumentou mais de 300\%(11).

$\mathrm{Na}$ última década, observou-se uma grande expansão dos cursos de pós-graduação lato sensu, como o de atenção primária à saúde e o de PSF. Esta ampliação de vagas ocorreu impulsionada pela Política Nacional de Atenção Básica e pela consequente expansão da Saúde da Família como estratégia de 
reorientação da APS.

Ademais, chama-se atenção para o alto percentual de enfermeiros do trabalho encontrado nesta pesquisa. Estudos indicam que o enfermeiro busca a especialização nesta área pela expectativa de atuar em diferentes ambientes que tenham a promoção da saúde como principal objetivo, assim como na saúde pública ${ }^{(14)}$.

Neste sentido, a região sudeste do Brasil, além de concentrar o maior número de instituições que oferecem o curso de especialização em Enfermagem do Trabalho, também possui o maior quantitativo de indústrias e empresas de pais ${ }^{(15)}$. Estas informações podem ser geradoras de uma forte expectativa em relação ao mercado de trabalho e retorno salarial, o que vem sendo um atrativo para os profissionais.

Sobre a desvinculação dos enfermeiros deste estudo com a ESF pode-se dizer que a saída dos profissionais gera uma ruptura, traduzindo-se em dificuldades de criação de vínculo com a comunidade, interrupção de programas de saúde e ações de educação permanente ${ }^{(16)}$

A descontinuidade pode ter sido provocada por duas questões. A primeira vincula-se ao fato de que alguns estudantes estavam vinculados ao Programa de Valorização do Profissional da Atenção Básica (PROVAB), cujo ciclo remuneratório foi interrompido no ultimo terço da formação desta turma. A segunda, pela grande rotatividade de profissionais relacionada ao tipo de vínculo trabalhista, visto que os trabalhadores estatutários correspondem a apenas $18 \%$ da força de trabalho(7).

O tempo médio de permanência de um enfermeiro na
ESF é de apenas um ano e a baixa permanência ocorre devido às modalidades flexíveis de contratação, que comumente se encontram à margem da legalidade ${ }^{(13)}$.

Foi observado neste estudo, um alto percentual de enfermeiros atuantes na ESF há no máximo cinco anos. Não se pode afirmar que a admissão recente esteja associada à alta rotatividade, entretanto, estima-se que este pode ser o resultado do aumento do número de equipes implantadas no estado nos últimos anos, que ultrapassa os $250 \%{ }^{(2)}$

Esse resultado encontra-se em consonância ao perfil de enfermeiros atuantes na ESF do Paraná, onde foi constatado que $80 \%$ dos enfermeiros possuem entre dois e cinco anos de trabalho ${ }^{(17)}$

\section{CONCLUSÃO}

Este estudo possibilitou a reflexão acerca de algumas consonâncias nacionais sobre o perfil dos enfermeiros atuantes na Estratégia de Saúde da Família.

Observou-se que o perfil de enfermeiros atuantes na estratégia de saúde da família sofreu modificações se comparado a décadas anteriores, principalmente a formação profissional, na qual evidenciou-se uma maciça vinculação de profissionais oriundos de instituições particulares de ensino.

Conclui-se que a ESF é um grande empregador das novas gerações da Enfermagem. A constância na ampliação da cobertura aponta para novos aumentos na empregabilidade, o que remete à necessidade da adequação das unidades de ensino para a formação de enfermeiros preparados para atuar na saúde coletiva.

\section{REFERÊNCIAS}

1. Mattos RA. O incentivo ao Programa de Saúde da Familia e seu impacto sobre as grandes cidades. Physis Rev Saúde Coletiva. 2002; 12(1): 77-108.

2. Ministério da Saúde (BR). Sistema Único de Saúde. Departamento de Atenção Básica. Evolução do credenciamento e DAB, 2015 implantação da ESF. 2015.[citado 2015 Ago 22]. Disponivel em: http://dab.saude.gov.br/ historico_cobertura_sf.php.

3. Faria MGA, David HMSL, Acioli S. Consultorias online: uma nova perspectiva no trabalho da enfermagem. Cogitare enferm. 2013; 18(2): 274-9.

4. GilAC. Métodos e técnicas de pesquisa social. 6.ed. São Paulo: Atlas; 2008.

5. Marconi MA, Lakatos EM. Fundamentos da metodologia científica. 5. Ed. São Paulo: Atlas; 2003.

6. Conselho Federal de Enfermagem. Núcleo de Estudos e Pesquisas de Recursos Humanos em Saúde. Perfil da Enfermagem do Brasil. 2015. [citado 2015 Ago 22]. Disponivel em: http://rj.corens.portalcofen.gov.br/wpcontent/uploads/2015/08/Apresentacao_Perfil_RIO-DE-JANEIRO.pdf.

7. Faria MGA, David HMSL, Acioli S. O perfil de enfermeiros atuantes na ESF em um Programa de Educação Permanente a Distância. Rev Enferm UERJ. 2012; 20: 591-5.

8. Correa ACP, Araujo EF, Ribeiro AC, Pedrosa ICF. Perfil sociodemográfico e profissional dos enfermeiros da atenção básica à saúde de Cuiabá - Mato Grosso. Rev Eletr Enf. 2012; 14(1): 172-80.

9. Tanabe LP, Kobayashi RM. Perfil, competências e fluência digital dos enfermeiros do Programa de Aprimoramento Profissional. Rev Esc Enferm. USP. 2013; 47(4): 943-9.
10. Gonçalves RMDA. Pedrosa LAP. Perfil dos enfermeiros da estratégia de Saúde da Familia e suas habilidades para atuar em saúde mental. Cienc Cuid Saude. 2009; 8(3): 345-51.

11. Ministério da Saúde (BR). Perfil dos médicos e enfermeiros do programa saúde da familia no Brasil: relatório final. Brasilia, DF. 2000. [citado 2015 Ago 22]. Disponivel em: http://bvsms.saude.gov.br/bvs/exposicoes/psf/ publicacoes/perfil_medico.pdf.

12. Organização Panamericana de Saúde. Panorama de la fuerza de trabajo en enfermeria en América Latina. Washington, DC: 2005. (Serie Desarrollo de Recursos Humanos n. 39). [citado 2015 Ago 22]. Disponivel em: <http:// www.ops.org.bo/textocompleto/ift26346.pdf>.

13. Ministério da Educação (BR).Sistema e-MEC.[citado 2015 Ago 22]. Disponivel em: http://emec.mec.gov.br/.

14. Paz PO, Kaiser DE. A busca pela formação especializada em enfermagem do trabalho por enfermeiros. Rev Gaúcha Enferm. 2011; 32(1): 23-30.

15. Zeitoune RCG, Oliveira DR, Montalvão EA, Muniz HD. A formação dos profissionais de enfermagem do trabalho na perspectiva das instituições formadoras. Rev Enferm UERJ. 2011; 19(3): 420-5.

16. Medeiros CRG, Junqueira AGW, Schwingel G, Carreno I, Jungles LAP, Saldanha OMFL. A rotatividade de enfermeiros e médicos: um impasse na implementação da Estratégia de Saúde da Familia. Ciênc Saúde Coletiva. 2010; 15(1): 1521-31

17. Ferrari RAP, Thomson Z, Melchior R. Estratégia da Saúde da Familia: perfil dos médicos e enfermeiros. Semina: Ciências Biológicas e da Saúde. 2005 26(2): 101-8 